\title{
Purification of a Low Molecular Weight Fucoidan for SPECT Molecular Imaging of Myocardial Infarction
}

\author{
Pierre Saboural ${ }^{1,2}$, Frédéric Chaubet ${ }^{1,2}$, Francois Rouzet ${ }^{1,3,4}$, Faisal Al-Shoukr ${ }^{1,3,4}$,

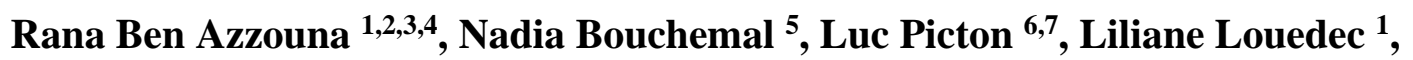 \\ Murielle Maire ${ }^{1,2}$, Lydia Rolland ${ }^{8}$, Guy Potier ${ }^{8}$, Dominique Le Guludec ${ }^{1,3,4}$, \\ Didier Letourneur ${ }^{1,2}$ and Cédric Chauvierre ${ }^{1,2, *}$
}

1 Inserm, U1148, LVTS, Paris Diderot University, Bichat-Claude Bernard Hospital, F-75877, Paris, France; E-Mails: pierre.saboural@univ-paris13.fr (P.S.); frederic.chaubet@ univ-paris13.fr (F.C.); francois.rouzet@bch.aphp.fr (F.R.); faisal1977@ hotmail.fr (F.A.-S.); rana.ben-azzouna@bch.aphp.fr (R.B.A.); liliane.louedec@inserm.fr (L.L.); murielle.maire@univ-paris13.fr (M.M.); dominique.leguludec@bch.aphp.fr (D.L.G.); didier.letourneur@inserm.fr (D.L.)

2 Galilée Institute, Paris 13 University, Sorbonne Paris Cité, F-93430, Villetaneuse, France

3 Multimodal Imaging Research Federation (FRIM), Paris Diderot University, F-75877, Paris, France

4 Nuclear Medicine Department, Bichat-Claude Bernard Hospital, AP-HP, F-75877, Paris, France

5 Laboratory CSPBAT, Paris 13 University, Sorbonne Paris Cité, CNRS UMR 7244, SBMB team, F-93017, Bobigny, France; E-Mail: nbouchemal@ smbh.univ-paris13.fr

6 Laboratory of Polymers Biopolymers Surfaces, Normandie University, Rouen University, F-76821, Mont Saint Aignan, France; E-Mail: luc.picton@ univ-rouen.fr

7 Laboratory of Polymers Biopolymers Surfaces, CNRS, UMR 6270 and FR3038, F-76821, Mont Saint Aignan, France

8 Algues \& Mer, Kernigou, F-29242, Ouessant, France;

E-Mails: lydia.rolland@algues-et-mer.com (L.R.); guy.potier@algues-et-mer.com (G.P.)

* Author to whom correspondence should be addressed; E-Mail: cedric.chauvierre@ inserm.fr; Tel.: +33-1-4025-7538; Fax: +33-1-4025-8602.

Received: 31 July 2014; in revised form: 5 September 2014 / Accepted: 9 September 2014 / Published: 23 September 2014

\footnotetext{
Abstract: Fucoidans constitute a large family of sulfated polysaccharides with several biochemical properties. A commercial fucoidan from brown algae, containing low molecular weight polysaccharidic species constituted of L-fucose, uronic acids and sulfate groups, was simply treated here with calcium acetate solution. This treatment led to a
} 
purified fraction with a yield of 45\%. The physicochemical characterizations of the purified fucoidan using colorimetric assay, MALLS, dRI, FT-IR, NMR, exhibited molecular weight distributions and chemical profiles similar for both fucoidans whereas the sulfate and L-fucose contents increased by $16 \%$ and $71 \%$, respectively. The biodistribution study in rat of both compounds labeled with ${ }^{99 \mathrm{~m}} \mathrm{Tc}$ evidenced a predominant renal elimination of the purified fucoidan, but the crude fucoidan was mainly retained in liver and spleen. In rat myocardial ischemia-reperfusion, we then demonstrated the better efficiency of the purified fucoidan. This purified sulfated polysaccharide appears promising for the development of molecular imaging in acute coronary syndrome.

Keywords: fucoidan; molecular imaging; atherothrombosis; SPECT

\section{Introduction}

Cardiovascular diseases (CVD) are the leading cause of death in the world [1] and medical imaging is the most widespread and efficient tool for their diagnosis. Molecular imaging is a recent and promising development of medical imaging dedicated to the visualization of the biological processes in vivo. However, the identification of mechanisms at cellular and molecular levels relies on the development of imaging probes that are able to interact with the targeted areas. The biospecificity of these probes is achieved by conjugation with small molecules [2,3], polymers and oligomers [4,5], antibodies, proteins and peptides [6,7], polysaccharides [8,9] or mixed compounds [10]. Fucoidans constitute a family of natural sulfated polysaccharides which have been extensively studied as they exhibit numerous biological activities: anticoagulant, antithrombotic [11], anti-inflammatory [12], anti-angiogenic [13] and anti-tumoral [14], anti-complementary [15,16] and anti-viral [12]. Recently, in purified systems and with human whole blood experiments, a low molecular weight fucoidan from brown algae was found as the most efficient glycosidic ligand of P-selectin, a glycoprotein expressed on the surface of activated platelets and on activated vascular endothelium [17]. This low molecular weight fucoidan was established as a relevant targeting agent for molecular imaging of atherothrombosis, either radiolabeled with technetium-99m $\left({ }^{99 \mathrm{~m}} \mathrm{Tc}\right)$ for Single Photon Emission Computed Tomography (SPECT) imaging [18] or bound to Ultrasmall Superparamagnetic Iron Oxide particles (USPIO) for Magnetic Resonance Imaging (MRI) [19].

Beyond the complete determination of the structures responsible for the interaction of fucoidan with P-selectin, the development of a biospecific probe for clinical molecular imaging of CVD is required to assess the overall fate of fucoidan within tissues and organs. Due to their natural origin and non-standardized methods of purification, fucoidans form a complex family of polysaccharides varying in molecular weight, structure and composition. In order to minimize this natural heterogeneity, previous studies from our laboratory have been performed with the same commercial fucoidan (Ascophyscient ${ }^{\circledR}$ ) provided by Algues and Mer [17-20]. This work presents a new specific treatment of fucoidan with calcium ions to obtain a purified extract. The molecular weight, structure and composition of the purified fucoidan were determined by physico-chemical techniques such as High Performance Size Exclusion Chromatography (HPSEC), Multi-Angle Laser Light Scattering 
(MALLS), viscosimetry, differential Refractive Index (dRI), colorimetric assays, Fourier Transform InfraRed spectroscopy (FT-IR), Nuclear Magnetic Resonance (NMR), and compared to those of the crude fucoidan. After radiolabeling with ${ }^{99 \mathrm{~m}} \mathrm{Tc}$ according to a previously described method [18], the biodistribution of both fucoidans was assessed in healthy rats. SPECT imaging was then performed in a model of ischemia-reperfusion (I-R) to evaluate the efficiency of the purified fucoidan for in vivo detection of ischemic events in rat myocardium.

\section{Results}

\subsection{Physico-Chemical Characterizations}

\subsubsection{Molecular Weight Measurement}

The purification treatment of crude fucoidan with aqueous calcium acetate led to a precipitate which was discarded by centrifugation. After dialysis and freeze-drying of the supernatant, purified fucoidan was obtained as a white fluffy powder in $45 \%$ average yield. The molecular weight measurements performed by HPSEC-MALLS-dRI showed two overlapping macromolecular populations (Figure 1): Light Scattering chromatograms showed one main peak with a shoulder at $27 \mathrm{~min}$. The molecular weight distribution was linear from 24 to $28.5 \mathrm{~min}$ and ranged from 26 to $1 \mathrm{kDa}$. The respective peak masses were M P1 $=9.0 \pm 0.1 \mathrm{kDa}$ and $\mathrm{MP} 2=3.5 \pm 0.1 \mathrm{kDa}$ for crude fucoidan and $\mathrm{M} P 1=8.8 \pm 0.1 \mathrm{kDa}$ and M P2 $=4.0 \pm 0.1 \mathrm{kDa}$ for purified fucoidan. The Refractive Index chromatogram of purified fucoidan did not present peaks after $30 \mathrm{~min}$, unlike crude fucoidan.

Figure 1. Refractive Index (plain line) and Light Scattering (dashed line) chromatograms and molecular mass profile (dotted line) of crude (Left) and purified (Right) fucoidans eluted in $0.1 \mathrm{M} \mathrm{LiNO}_{3}$ at $0.5 \mathrm{~mL} / \mathrm{min}$ with Shodex SB-802.5 and SB-803 columns.
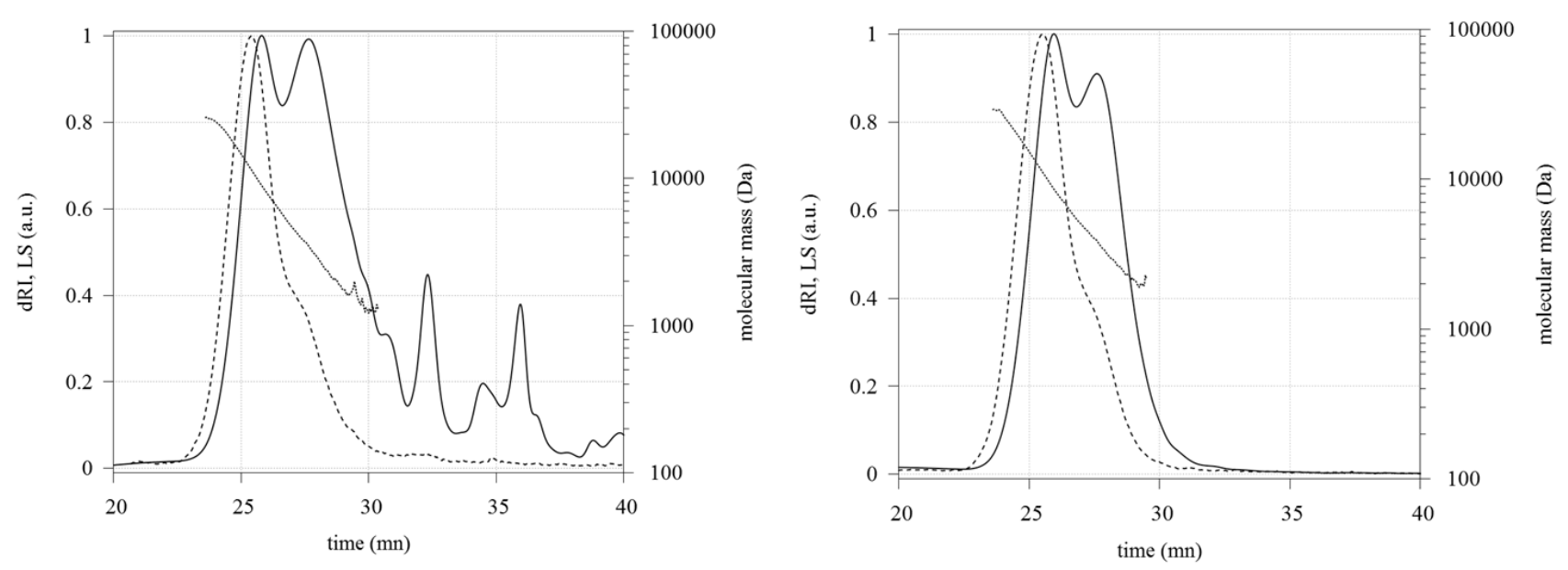

Average number $\left(\mathrm{M}_{\mathrm{n}}\right)$ and average weight $\left(\mathrm{M}_{\mathrm{w}}\right)$ molecular weights of purified fucoidan were increased compared to crude fucoidan but the polydispersity was not modified (Table 1), $\mathrm{M}_{\mathrm{w}}$ being less affected by the purification, that is consistent with the elimination of small molecules by the purification process. 
Table 1. Molecular weights of crude and purified fucoidans.

\begin{tabular}{cccc}
\hline Fucoidans & $\overline{\boldsymbol{M}_{\boldsymbol{n}}}(\mathbf{k D a})$ & $\overline{\boldsymbol{M}_{\boldsymbol{w}}}(\mathbf{k D a})$ & $\overline{\boldsymbol{M}_{\boldsymbol{n}}} / \overline{\boldsymbol{M}_{\boldsymbol{w}}}$ \\
\hline Crude & $3.5 \pm 0.4$ & $6.2 \pm 0.3$ & $1.5 \pm 0.2$ \\
Purified & $4.9 \pm 0.2$ & $7.5 \pm 0.2$ & $1.5 \pm 0.1$ \\
\hline
\end{tabular}

\subsubsection{Chemical Composition}

L-fucose, uronic acid and sulfate contents assayed by colorimetric methods were gathered in Table 2 . Crude fucoidan was mainly composed of L-fucose and sulfate with a lower amount of uronic acid. After the purification step with calcium acetate, purified fucoidan was enriched in L-fucose and sulfate by $71 \%$ and $16 \%$, respectively. Moreover, the proportion of other components in fucoidan samples decreased from $37.2 \%$ to $15.2 \%$.

Table 2. Composition of the fucoidan samples (w/w \% of dried weight).

\begin{tabular}{ccccc}
\hline Fucoidans & L-Fucose & Uronic Acid & Sulfate & Other \\
\hline Crude & $25.0 \% \pm 0.5 \%$ & $16.1 \% \pm 0.9 \%$ & $21.7 \% \pm 2.0 \%$ & $37.2 \%$ \\
Purified & $42.8 \% \pm 2.2 \%$ & $16.9 \% \pm 0.4 \%$ & $25.1 \% \pm 0.8 \%$ & $15.2 \%$ \\
\hline
\end{tabular}

\subsubsection{FTIR Analysis}

FT-IR spectra of crude and purified fucoidans (Figure 2) presented similar patterns corresponding to that of sulfated polysaccharides [21-23], a broad band at $3450 \mathrm{~cm}^{-1}$ (O-H groups stretching), two small sharp bands at $2940 \mathrm{~cm}^{-1}$ and $2885 \mathrm{~cm}^{-1}$ (C-H stretching of the pyranose ring and of the methyl group of fucose), two bands at $1600 \mathrm{~cm}^{-1}$ and $1420 \mathrm{~cm}^{-1}$ (COOH stretching vibration), a band around $1250 \mathrm{~cm}^{-1}$ ( $\mathrm{S}=\mathrm{O}$ stretching) and two small bands at $840 \mathrm{~cm}^{-1}$ and $820 \mathrm{~cm}^{-1}$ (axial and equatorial C-O-S bending, respectively).

Figure 2. FT-IR spectra of crude (plain line) and purified (dashed line) fucoidans. Arrows indicate the characteristic bands for sulfated polysaccharides.

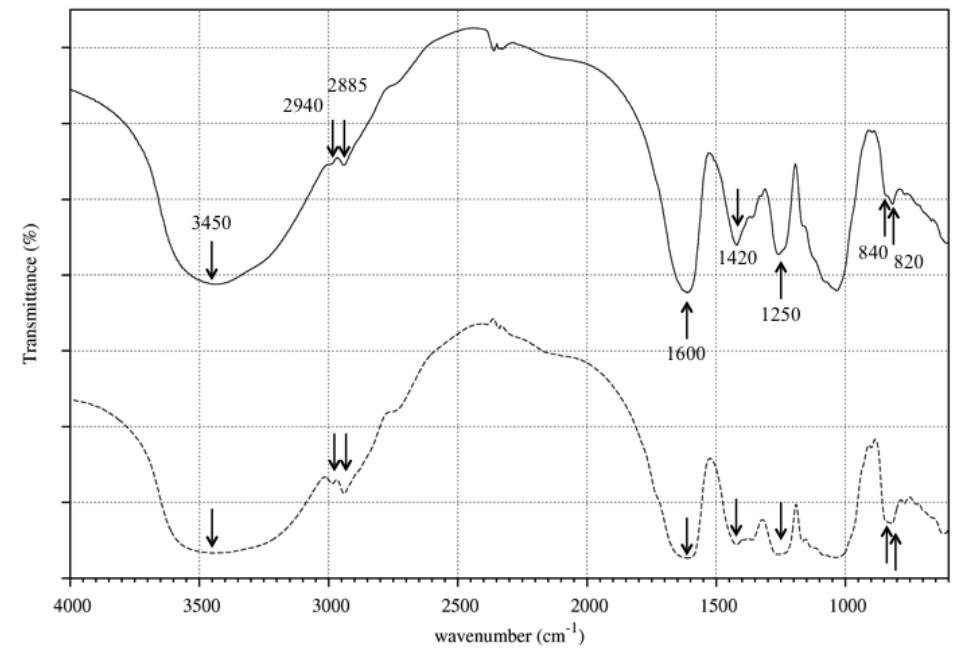




\subsubsection{NMR Analysis}

NMR spectra of both compounds also presented the same general features (Figure 3). Signals from anomeric protons $\mathrm{H}-1$ arose from $\alpha$-L-fucose residues and uronic acid residues between 5.6 and 5.15 ppm. H-2, H-3 and H-4 of the sulfated fucose could be localized between 4.66 and 4.10 ppm whereas from 4.20 to 3 ppm were H-2, H-3, H-4 and H-5 of unsulfated fucose and uronic acid. Lastly, the two peaks, around 1.2 and $1.35 \mathrm{ppm}$ were assigned to the methyl group of fucose. A single peak at $2.2 \mathrm{ppm}$ might be ascribed to acetyl groups.

Figure 3. ${ }^{1} \mathrm{H}-\mathrm{NMR}$ spectra (in $99.8 \% \quad \mathrm{D}_{2} \mathrm{O}, 500 \mathrm{MHz}$ ) of crude (upper) and purified (lower) fucoidans. (A) Anomeric protons from $\alpha$-L-Fucose; (B) H-2, H-3 and H-4 of the sulfated fucose; (C) H-2, H-3, H-4 and H-5 of unsulfated fucose and uronic acid; (D) Methyl protons.

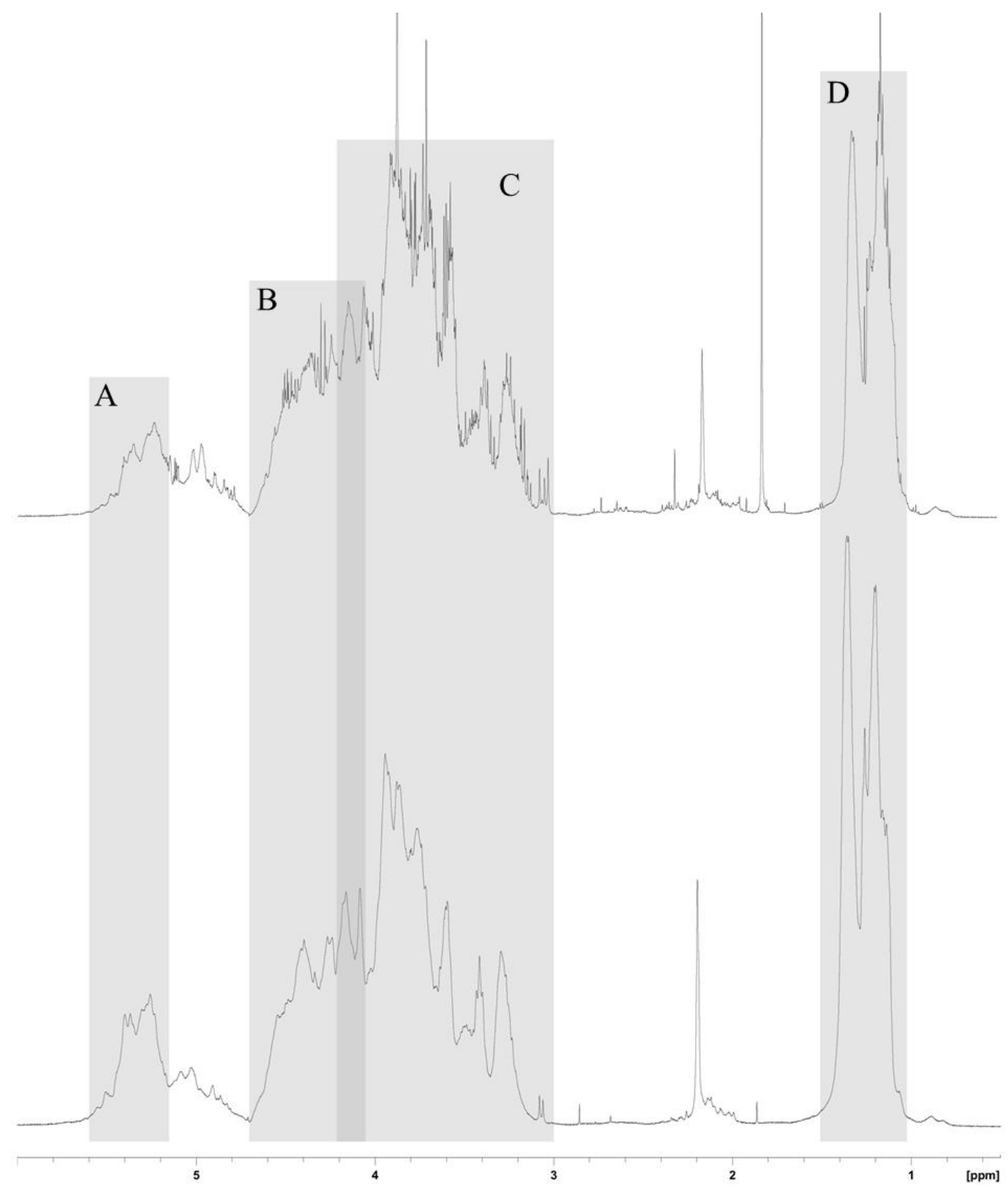




\subsection{In Vivo Studies of the ${ }^{99 m}$ Tc-Radiolabeled Fucoidans}

The radiolabeling of crude and purified fucoidans was performed with ${ }^{99 \mathrm{~m}} \mathrm{Tc}$ according to a previously described procedure [18]. The radiochemical purity was superior to $95 \%$ for both fucoidans without any significant difference.

\subsubsection{Biodistribution Studies of the Radiolabeled Fucoidans}

The uptake of ${ }^{99 \mathrm{~m}} \mathrm{Tc}$-crude fucoidan was intense in the rat liver and only faint in the bladder and kidneys (Figure 4, left panel). In contrast, the uptake of purified fucoidan was faint in the liver and intense in both bladder and kidneys (Figure 4, right panel). Quantifications of uptake ratios obtained by SPECT are presented in Figure 5. No free ${ }^{99 \mathrm{~m}} \mathrm{Tc}$ was detected in thyroid for both compounds indicating a stable complexation even after $2 \mathrm{~h}$ in vivo. Note that the background signal of the body was higher for purified fucoidan than for crude fucoidan since the images were normalized on the highest pixel signal (in white on the color images).

Figure 4. Representative whole body SPECT images of the ${ }^{99 \mathrm{~m}}$ Tc-radiolabeled fucoidans: crude (left) and purified (right). The rats (five for crude fucoidan and four for purified fucoidan) were imaged $2 \mathrm{~h}$ after injection of $2 \mu \mathrm{g}$ of the samples.

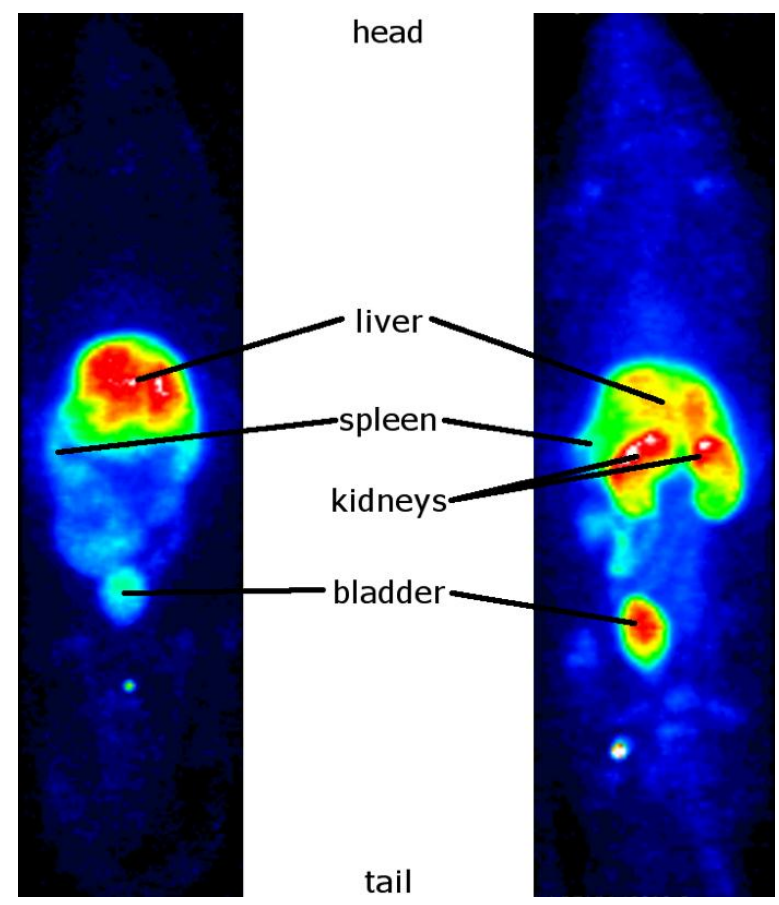


Figure 5. Quantification of the biodistribution of crude fucoidan (grey) and purified fucoidan (black) based on the whole body SPECT images obtained $2 \mathrm{~h}$ after i.v. injection of the ${ }^{99 \mathrm{~m}} \mathrm{Tc}$-radiolabeled fucoidans in rats (respectively $n=5$ and $n=4$ ) expressed as mean \pm SD. Three Regions of Interest (ROI) were assessed: the hepatosplenic system (liver + spleen), the urinary system (kidneys + bladder) and the other parts (the hepatosplenic system and the urinary system activities subtracted from whole body activity). ${ }^{*} p<0.05$.

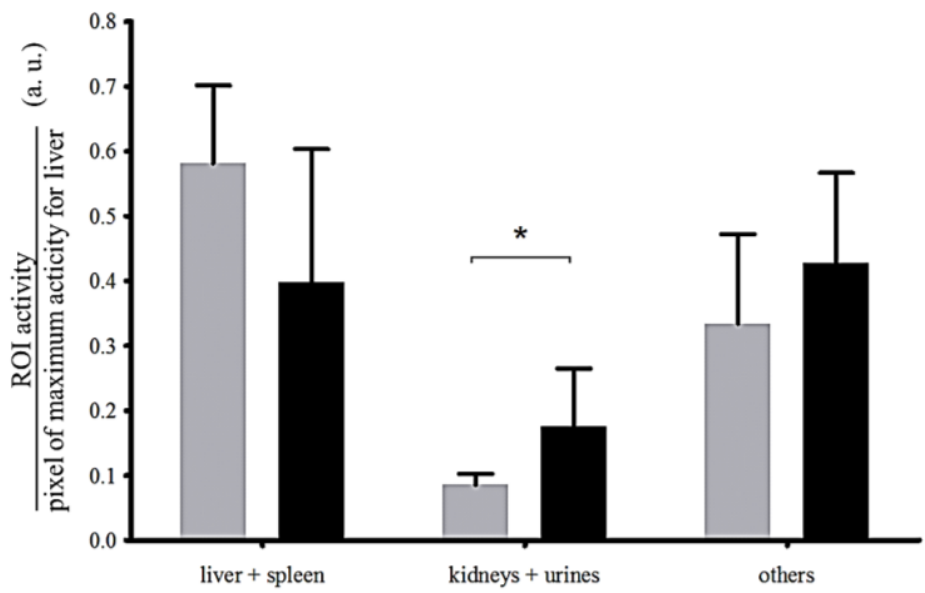

\subsubsection{Myocardial Ischemia-Reperfusion}

In the model of myocardial Ischemia-Reperfusion in rat, purified fucoidan facilitated the in vivo detection of the ischemic myocardium with a signal intensity twice that of the crude form (Figure 6). A similar finding was obtained by autoradiography (Figure 7). The ratio of the injured tissue (dark area) activity over the normal myocardium was computed for crude fucoidan $(4.9 \pm 0.2)$ and for purified fucoidan $(8.7 \pm 2.1)$.

Figure 6. SPECT/CT (from left to right: sagittal, coronal and axial plane) of myocardial ischemia-reperfusion in rat, acquired $2 \mathrm{~h}$ after the injection of the radiolabeled crude fucoidan (A) and purified fucoidan (B). The SPECT color-scale was normalized (max: 20 counts/pixel). The focal uptake of the tracer was localized in the area of ischemia provoked in the left ventricle (white arrow), with a myocardium to background (blood pool) ratio of 1.9 with the crude fucoidan and 3.3 with the purified form.

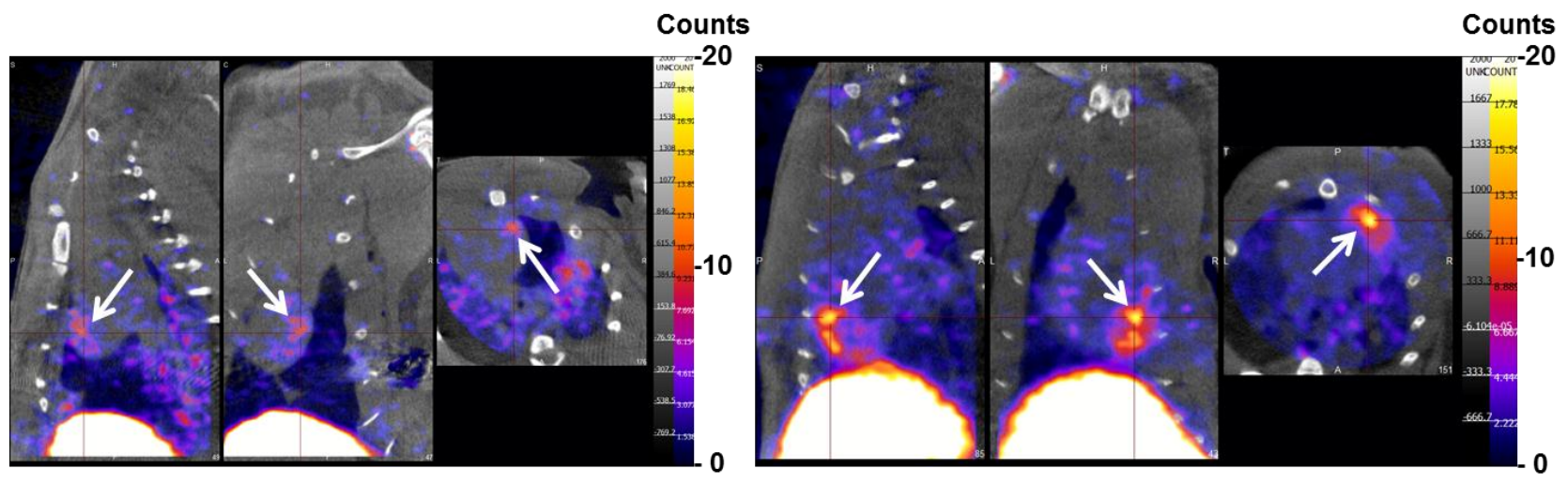


Figure 7. Autoradiography ( $20 \mu \mathrm{m}$ thickness transverse slices) of the myocardium after injection of the radiolabeled crude fucoidan (A) and purified fucoidan (B) in an I-R rat model. The uptake of the radiolabeled fucoidans appears in black.

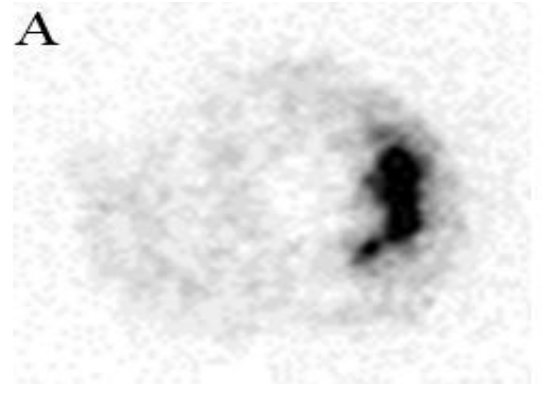

B

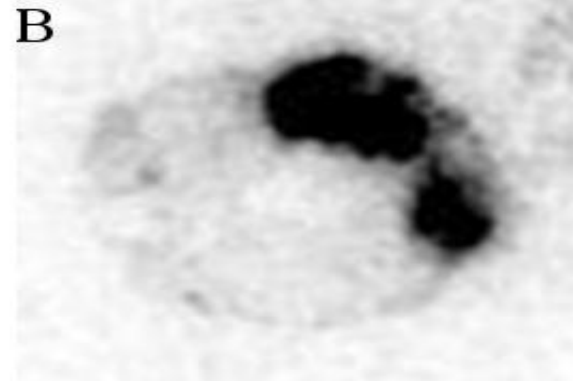

\section{Discussions}

Fucoidans are interesting bioactive sulfated polysaccharides to be developed for clinical purposes. They are endowed with numerous biological activities, are cheap and their preparations from marine algae allow protocols compatible with industrial constraints without costly specific equipment. However, there is currently no standard method to obtain a bioactive well defined fucoidan from crude carbohydrate extracts. Fucoidans are always obtained as mixtures of macromolecular species, the structures of which depend on many parameters, such as the algal species or the period and location of harvesting and the purification methods $[24,25]$. Commercial products with the same identification are for instance highly variable in their molecular weight (from 20 to $170 \mathrm{kDa}$ ) and composition.

Numerous studies have been published on structure-activity relationships of fucoidans from marine algae [12,26] since this species was identified by Kylin one century ago [27]. Fucoidans belong to the family of fucans including two other carbohydrate species: ascophyllans and sargassans [28]. Fucoidans refer to polysulfated poly-L-fucose, although xylose, glucose, galactose or glucuronic acids can be associated [29], as ascophyllans and sargassans are uronic acid-rich and galactose-rich polysaccharidic species, respectively. Uronic acids are frequently recovered in fucoidans from algae either as co-extracted polysaccharidic structures, or as discrete short branchings [25]. Eventually, repeated simple fucoidan structures can be obtained but always after several purification steps from crude fucoidan extracts [30-33]. To date, only sulfated fucose rich species have been reported to be responsible for the biological properties of fucoidans [11,12]. Recent studies demonstrated that Ascophysicent ${ }^{\circledR}$ (crude fucoidan), a commercially available low molecular weight fucoidan extracted from Ascophyllum nodosum, is a relevant biomarker for the molecular imaging of thrombus and vascular activation in atherothrombotic diseases [18,19]. However, sulfate and fucose represent less than 50 percent of the dry weight of crude fucoidan with a remaining high proportion of uronic acids and other sugars. It is likely that it contains a mixture of bioactive sulfated fucose-based polymers and some polyuronic acids, likely ascophyllan. This work aimed at (i) purifying crude fucoidan by precipitating the polyuronic structures with calcium ions; (ii) compare biodistribution of crude $v$ s. purified fucoidan with regards to the route of blood clearance and (iii) demonstrate that the purification process did not alter radiolabeling efficiency and in vivo binding.

The purification with calcium ions was intended to remove uronic acid chains contained in the crude fucoidan. As the alginates are expected to be removed by the industrial extraction process (data not 
shown), it has been thought that the uronic acids came from the ascophyllans, a sulfated polysaccharide with an uronic acid backbone and sulfated polyfucose branchings [34,35], that could be co-extracted with the fucoidan. The uronic acid backbone of ascophyllans would form complexes with calcium ions and then precipitate. Our findings showed that the FT-IR and ${ }^{1} \mathrm{H}-\mathrm{NMR}$ spectra of crude and purified fucoidans presented typical features of a sulfated polysaccharide with sulfation at O-2, O-3 and O-4 positions of L-fucose as previously demonstrated with fucoidan extracts from A. nodosum [22,36]. Compared to other fucoidans extracted from A. nodosum, crude fucoidan is composed of two low molecular weight species: one with a lower fucose rate and the other with a higher uronic acid rate $[29,37,38]$. By comparing the quantity of uronic acids to the total quantity of fucose and sulfate groups of crude and purified fucoidans-34.5\% and $24.9 \%$, respectively-it appeared that around $10 \%$ of uronic acids were removed by the calcium treatment. Moreover, the molecular weight distribution of purified fucoidan was shifted to slightly higher molecular weights as small molecules were removed.

The radiolabeling with ${ }^{99 \mathrm{~m}} \mathrm{Tc}$ was achieved for both species with a radiochemical purity higher than $95 \%$ similar to that of the ${ }^{99 \mathrm{~m}} \mathrm{Tc}$-heparin [39]. In vivo, ${ }^{99 \mathrm{~m}} \mathrm{Tc}$-crude fucoidan was localized in part in the liver and the spleen which are the targeted organs of the reticulo-endothelial system (RES). This in vivo data is interesting since low molecular weight fucoidan from A. nodosum was reported to have in vitro anti-complement activity [16]. The higher accumulation of ${ }^{99 \mathrm{~m}} \mathrm{Tc}$-crude fucoidan in the liver and spleen compared to that of ${ }^{99 \mathrm{~m}} \mathrm{Tc}$-purified fucoidan could be due to the higher quantity of uronic acids.

Finally, the purification procedure did not affect in vivo binding of fucoidan to endothelial cells activated by a transient ischemic event. In the Ischemic-Reperfusion rat model, focal uptakes of the polysaccharides were detectable for both ${ }^{99 \mathrm{~m}} \mathrm{Tc}$-crude fucoidan and ${ }^{99 \mathrm{~m}} \mathrm{Tc}$-purified fucoidan. Interestingly, it was more intense for the purified fucoidan and the autoradiographies strengthened this observation.

\section{Experimental Section}

\subsection{Polysaccharide and Common Chemicals}

Fucoidan was obtained from Algues \& Mer (Ascophycient ${ }^{\circledR}$, batch \#ASPHY12399, Ouessant, France), DEAE-cellulose resin from Sigma Aldrich and dialysis membrane from Spectra/Por, (MWCO $1 \mathrm{kDa}$, Dominique Dutscher, France). Common chemicals were purchased in analytical grade from Carlo Erba, Sigma-Aldrich and Fisher Scientific and used without further purifications.

\subsection{Molecular Weight Determination}

The absolute average molecular weights and molecular weight distributions were determined at room temperature by coupling online a high performance size exclusion chromatograph (HPSEC), a multi-angle laser light scattering detector (MALLS), a viscometer and a differential refractive index (dRI) detector. 0.1 $\mathrm{M} \mathrm{LiNO}_{3}$, used as carrier, was filtered through a $0.1 \mu \mathrm{m}$ filter unit (Millipore, Billerica, USA), carefully degassed (DGU-20A3R Shimadzu, Kyoto, Japan), and eluted at a $0.5 \mathrm{~mL} / \mathrm{min}$ flow rate (LC10Ai Shimadzu, Kyoto, Japan). $100 \mu \mathrm{L}$ of a $0.45 \mu \mathrm{m}$-filtered sample solution (at about $20 \mathrm{mg} / \mathrm{mL}$ ) were injected with an automatic injector (SIL-20A HT Shimadzu, Kyoto, Japan). The SEC line consisted of an OHpak SB-G guard column for protection and two OHpak SB-802.5 and-803 HQ columns (Showa Denko Europe, Munich, Germany) in series. The column packing was a poly 
(hydroxymethacrylate) gel. The MALLS photometer, a DAWN HELEOS II from Wyatt Technology Inc. (Santa Barbara, CA, USA) was provided with a fused silica cell and a Ga-As laser $(\lambda=665.8 \mathrm{~nm})$. The viscometer was a ViscoStar II from Wyatt Technology Inc. (Santa Barbara, CA, USA). The whole collected data: light scattering (LS), dRI and viscosity were analyzed using the Astra v6.0.6 software package. Molar mass were obtained with the Zimm order 1 method using angles between $53.1^{\circ}$ and $140^{\circ}$. The concentration of each eluted fraction was determined with dRI (RID10A Shimadzu, Kyoto, Japan) according to the measured values of $d n / d c(0.144 \mathrm{~mL} / \mathrm{g})$. The determination of the average intrinsic viscosity allowed us to obtain the average hydrodynamic volume $\left(\mathrm{V}_{\mathrm{h}}\right.$, i.e., the average hydrodynamic radius $\mathrm{R}_{\mathrm{h}}$ ) using the Einstein-Simha equation: $V_{h}=\frac{[\eta] \cdot M}{v \cdot N_{A}}$ where $\mathrm{N}_{\mathrm{A}}$ is Avogadro's number, $\mathrm{M}$ is the molar mass, $[\eta]$ is the intrinsic viscosity $(\mathrm{g} / \mathrm{mL})$, and $v$ is a conformational parameter that takes the value of 2.5 (for spherical random coil).

\subsection{Purification of Fucoidan}

The purification of fucoidan was achieved after dissolution $(160 \mathrm{mg} / \mathrm{mL})$ of crude fucoidan with aqueous calcium acetate $(20 \mathrm{mM})$ during $4 \mathrm{~h}$ at $55{ }^{\circ} \mathrm{C}$ under magnetic stirring and the $\mathrm{pH}$ was constantly adjusted between 6.5 and 7.5. The solution was left at $4{ }^{\circ} \mathrm{C}$ overnight and then centrifuged $15 \mathrm{~min}$ at $4500 \mathrm{rpm}$ (Allegra X-15R centrifuge, Beckman-Coulter France, Villepinte, France). The supernatant was dialyzed against water (MWCO 1 kDa, Dominique Dutscher, France) and freeze-dried.

\subsection{Colorimetric Assay}

The colorimetric assay was achieved on microplate and ELx800 Absorbance Microplate Reader (BioTek, Colmar, France) and processed with Gen-5 software (BioTek, Colmar, France).

\subsubsection{Fucose}

The fucose rate determination was adapted from the Dische method [40]. In a 96-wells plate, $50 \mu \mathrm{L}$ of sample (about $0.3 \mathrm{mg} / \mathrm{mL}$ ) was mixed with $200 \mu \mathrm{L}$ of sulfuric acid $(18 \mathrm{M})$. The plate was heated during $30 \mathrm{~min}$ at $80{ }^{\circ} \mathrm{C}$ then left at room temperature for cooling down. $8 \mu \mathrm{L}$ of L-cysteine hydrochloride at $3 \%(\mathrm{w} / \mathrm{v})$ was added and left to react for $1 \mathrm{~h}$ at $4{ }^{\circ} \mathrm{C}$. Then absorbance was computed as follows: $\mathrm{OD}_{\text {fucose }}=\left[\mathrm{OD}_{405}(\right.$ sample $)-\mathrm{OD}_{405}$ (blank) $]-\left[\mathrm{OD}_{450}\right.$ (sample) $-\mathrm{OD}_{450}$ (blank) $]$. The amount of fucose was determined from a standard curve obtained with L-fucose solutions $(25-500 \mu \mathrm{g} / \mathrm{mL})$.

\subsubsection{Glucuronic Acid}

Glucuronic acid rate determination was adapted from Bitter et al. [41]. In a 96-wells plate, $35 \mu \mathrm{L}$ of sample (about $0.3 \mathrm{mg} / \mathrm{mL}$ ) was mixed with $200 \mu \mathrm{L}$ of sodium tetraborate $(0.025 \mathrm{M})$ in sulfuric acid $(18 \mathrm{M})$. The plate was heated during $30 \mathrm{~min}$ at $80{ }^{\circ} \mathrm{C}$ then left at room temperature for cooling down. $14 \mu \mathrm{L}$ of carbazol solution at $0.15 \%(\mathrm{w} / \mathrm{v})$ in ethanol was added and heated $1 \mathrm{~h}$ at $80{ }^{\circ} \mathrm{C}$, and then the absorbance was read at $540 \mathrm{~nm}$. The amount of uronic acid was determined from a standard curve obtained with D-glucuronic acid solutions submitted to the same process. 


\subsubsection{Sulfate}

Sulfate rate was obtained by formation of methylene blue after acidic hydrolysis of the samples, reduction of sulfate as hydrogen sulfide $\left(\mathrm{H}_{2} \mathrm{~S}\right)$, and formation of methylene blue from $N, N$-dimethyl phenylene diamine dihydrochloride in strong acidic medium in presence of ferric chloride. Conditions were optimized from Gustafsson [42] and Kuban et al. [43]. Briefly, 100-200 $\mu \mathrm{L}$ of the sample were added to $5 \mathrm{~mL}$ of a reducing mixture prepared with $100 \mathrm{~mL}$ of concentrated hydroiodic acid, $25 \mathrm{~mL}$ of glacial acetic acid and $2.5 \mathrm{~g}$ of sodium hypophosphite. The mixture was refluxed during $20 \mathrm{~min}$ through a water-cooled condenser under a stream of $\mathrm{N}_{2}(100 \mathrm{~mL} / \mathrm{min})$ which carried away evolved $\mathrm{H}_{2} \mathrm{~S}$. After bubbling through a gas-washing column $(20 \mathrm{~mL}$ of Tris buffer $0.1 \mathrm{M}, \mathrm{pH} 7.2), \mathrm{H}_{2} \mathrm{~S}$ was trapped as zinc sulfide $(\mathrm{ZnS})$ in $30 \mathrm{~mL}$ of a solution of zinc acetate prepared by diluting $5 \mathrm{~mL}$ at $0.5 \mathrm{M}$ and sodium acetate $0.1 \mathrm{M}$ with $25 \mathrm{~mL}$ of deionized water. Eight $\mathrm{mL}$ of $16 \mathrm{mM}$ ferric chloride in $\mathrm{H}_{2} \mathrm{SO}_{4}, 0.1 \mathrm{M}$, and $2 \mathrm{~mL}$ of $3.7 \mathrm{mM} \mathrm{N}, N$-dimethyl-phenylene-diamine dihydrochloride in $\mathrm{H}_{2} \mathrm{SO}_{4}, 9 \mathrm{M}$ were added to the $\mathrm{ZnS}$ solution, and the final volume was adjusted to $50 \mathrm{~mL}$ with deionized water. The vial was maintained at room temperature in the dark for $20 \mathrm{~min}$ and the absorption was measured at $665 \mathrm{~nm}$ with a UV-visible spectrophotometer (Lambda 12, Perkin-Elmer, Courtaboeuf, France). The amount of sulfur was determined from a standard curve obtained with potassium sulfate solutions submitted to the overall process. This method did not require a special treatment of the samples and was not sensitive to ferric ions contrary to sulfur elemental analysis or turbidimetric assays.

\subsection{Infrared Spectra}

Infrared spectra were acquired between 400 and $4000 \mathrm{~cm}^{-1}$ with an AVATAR 370 FT-IR spectrometer (Thermo-Nicolet, Villebon, France) with 32 scans/sample and a resolution of $2 \mathrm{~cm}^{-1}$. Dry samples were pressed with potassium bromide $(2 \% \mathrm{w} / \mathrm{w})$. Spectra were processed and analyzed with OMNIC v6.1 (Thermo-Nicolet software).

\subsection{Nuclear Magnetic Resonance (NMR)}

All NMR experiments were conducted on a Bruker AVANCE III spectrometer (BioSpin Bruker, Wissembourg, France) operating at a proton frequency of $500 \mathrm{MHz}$ with a $5 \mathrm{~mm}$ gradient indirect detection probe, at a probe temperature of $300 \mathrm{~K}$. The samples were exchanged twice with $99.8 \% \mathrm{D}_{2} \mathrm{O}$ with intermediate freeze drying and dissolved in $0.6 \mathrm{~mL}$ of $99.96 \% \mathrm{D}_{2} \mathrm{O}$. 1-D proton spectra were acquired with 16 scans and $32 \mathrm{~K}$ data points with a spectral width of $5000 \mathrm{~Hz}$. Typical ${ }^{1} \mathrm{H} 9.5 \mu$ s-pulse length and relaxation delay of $1 \mathrm{~s}$ were used. Water signal was suppressed by a presaturation sequence at the water signal frequency.

\subsection{Radiolabeling Procedure}

The radiolabeling of fucoidan has been performed according to the general process of reduction of pertechnetate $\left(\mathrm{TcO}_{4}{ }^{-}\right)$by stannous ions in order to obtain a complex between a reduced form of ${ }^{99 \mathrm{~m}} \mathrm{Tc}$ and the ligand. The procedure is the most widely used to produce ${ }^{99 \mathrm{~m}} \mathrm{Tc}$-labeled radiopharmaceuticals both in the preclinical and clinical domains [44]. The mechanism of complexation generally accepted involves the formation of positively charged reduced forms of ${ }^{99 \mathrm{~m}} \mathrm{Tc}[\mathrm{Tc}(\mathrm{V})$ and/or $\mathrm{Tc}(\mathrm{III})]$ that will 
react with negatively charged domains of the ligand [44]. This procedure has already been applied to radiolabeling of polysaccharides such as heparin [45] and dextran [46]. Briefly, ${ }^{99 \mathrm{~m}} \mathrm{Tc}$ was eluted from a ${ }^{99} \mathrm{Mo} /{ }^{99 \mathrm{~m}} \mathrm{Tc}$ generator as an isotonic solution with high specific activity. The sodium pertechnetate $\left({ }^{99 m} \mathrm{TcO}_{4} \mathrm{Na}\right.$, between 300 and $400 \mathrm{MBq}$ in $<200 \mu \mathrm{L}$ saline, freshly eluted), $1 \mu \mathrm{g}$ stannous chloride $(1 \mu \mathrm{g} / \mu \mathrm{L}$; Sigma-Aldrich, Saint-Quentin Fallavier, France) and $1 \mu \mathrm{g}$ potassium borohydride $(1 \mu \mathrm{g} / \mu \mathrm{L}$; Sigma-Aldrich, Saint-Quentin Fallavier, France) were added to a vial containing $10 \mu \mathrm{g}$ of fucoidan $(1 \mathrm{mg} / \mathrm{mL})$, and left to incubate during $1 \mathrm{~h}$ at room temperature. The quality control was performed with instant thin-layer chromatography (ITLC) developed in methyl-ethyl-ketone buffer. The radiochemical purity was always superior to $95 \%$. No supplementary purification was performed on the radiolabeled fucoidan.

\subsection{Biodistribution and Experimental Model of Myocardial Ischemia-Reperfusion}

For both the biodistribution and ischemia-reperfusion rat model, each rat was injected with $200-500 \mu \mathrm{L}$ of $60-74 \mathrm{MBq}$ of radiolabeled fucoidan, corresponding to about $2 \mu \mathrm{g}$ of fucoidan and the injected dose $\mathrm{pH}$ was neutral (7 and 7.5). The rats weighted between 310 and $350 \mathrm{~g}$.

Biodistribution of radiolabeled fucoidans was performed in healthy male Wistar rats (Janvier, France) in order to determine the preferential route of blood clearance after intravenous injection. Since we previously determined that blood clearance of fucoidan was maximal during the first $2 \mathrm{~h}$ after administration [18], we set the delay between injection and biodistribution assessment at $2 \mathrm{~h}$. We determined in vivo biodistribution of fucoidans by SPECT imaging which is inherently quantitative and is now widely used for this purpose.

Whole body acquisitions were performed under isoflurane anesthesia after intravenous injection (penile vein) of $50 \mathrm{MBq}$ of crude $(n=5)$ or purified $(n=4)$ fucoidans. Total body activity was estimated using a volume of interest encompassing the whole field of view, subtracted from residual activity remaining at the injection site (penis). Then, relative organ retention (defined for liver, spleen, left kidney, and bladder) was calculated as the ratio of activity within 3-dimensional volumes of interest over total body activity. Results were then displayed as renal excretion (kidney $\times 2+$ bladder) and reticulo-endothelial retention (liver + spleen).

The model of myocardial ischemia-reperfusion was performed in four male Wistar rats. The proximal left anterior descending coronary artery was occluded using a suture around a catheter for $20 \mathrm{~min}$, then was reperfused by cutting the suture along the catheter in rats under general anesthesia (ketamine/xylazine) and positive pressure ventilation. Injection of the radiotracer was performed after $2 \mathrm{~h}$ of reperfusion. This study was conducted under authorization of the French Direction of the Veterinary Services (No. 2012-15/698-106).

\subsection{Imaging Procedures}

\subsubsection{Acquisition and Reconstruction Parameters}

Imaging was performed $2 \mathrm{~h}$ after intravenous injection of radiolabeled fucoidans. Helical SPECT/CT scans were performed under isoflurane anesthesia using a 4-headed multiplexing multipinhole camera (NanoSPECT/CT plus, Bioscan Inc., Washington, DC, USA). Each head was 
equipped with a tungsten collimator (Rat Whole Body-High sensitivity). Flat-panel detector CT was performed first (tube voltage: $55 \mathrm{kV}$, tube current: $145 \mathrm{mAs}$ ), then whole body SPECT acquisition was performed with the following parameters: helical scan with 28 projections/rotation plus circular scan at the beginning and at the end of the scan range, matrix size: $256 \times 256$, zoom: 1.14 (pixel size: $1 \mathrm{~mm}^{2}$ ). SPECT data were reconstructed using the HiSPECT (Bioscan Inc., Washington, DC, USA) iterative reconstruction software on a dedicated workstation, and visualized using InVivoScope software with co-registration of SPECT and CT images.

\subsubsection{Data Analysis}

Radiolabeled fucoidan biodistribution in healthy rats was carried out in order to establish evidence of the preferential route of blood clearance after intravenous injection. Total body activity was estimated using a volume of interest encompassing the whole field of view, subtracted from residual activity remaining at the injection site (penis). Then, relative organ retention (defined for liver, spleen, left kidney, and bladder) was calculated as the ratio of activity within 3-dimensional volumes of interest over total body activity. Results were then displayed as renal excretion (kidney $\times 2+$ bladder) and reticulo-endothelial retention (liver + spleen).

Ischemia-reperfusion model scintigrams were assessed visually to determine the presence of a focal tracer uptake in the myocardium.

Autoradiography: after completion of SPECT/CT, animals were euthanized with pentobarbital overdose. Tissue samples were frozen and cut into transverse sections of $20 \mu \mathrm{m}$ thickness which were exposed in a digital $\beta$-imager (Beta Imager, Biospace Lab, Paris, France) for 6 h. Quantification was performed by calculating the ratio between the activity (mean counts $/ \mathrm{mm}^{2}$ ) of the area at risk and the activity of a region of interest drawn over normal myocardium.

\subsection{Statistical Analyses}

Statistics were computed with GraphPad Prism v.5 software. Chemical compositions of the samples were compared with a two-tailed $t$-test where $p<0.05$ was considered significant. For biodistribution, each sample was compared 2 by 2 in a ROI using a two-tailed Mann-Whitney test and was considered significant at $p<0.05$.

\section{Conclusions}

Ascophyscient ${ }^{\circledR}$ is a commercially available product extracted from Ascophyllum nodosum mainly composed of fucose, sulfate and uronic acids. After a simple purification step, its composition was enriched in sulfated fucose. The biodistribution of the purified fucoidan in healthy rats was improved ensuring that the renal elimination was favored. Finally, the binding of ${ }^{99 \mathrm{~m}} \mathrm{Tc}$-purified fucoidan to the activated endothelium was validated in rat model of myocardial ischemia. As a low cost and easily labeling ligand of ischemic events, this purified fucoidan is promising for the clinical development of a molecular imaging probe for myocardial infarctions. 


\section{Acknowledgments}

This study was supported by Inserm, University Paris 13 and the competitiveness cluster Medicen Paris Region. P.S. is a recipient of the grant from University Paris 13 and IMOVA project founded by FUI/OSEO. The authors would like to thank Thomas Cognet and Fadwa Aït-Ali for their technical assistance, Algues \& Mer for the supplying of fucoidan, and Richard Bayles for editing of the manuscript. We also acknowledge the financial supports from FP7 NMP-LA-2012-309820 "NanoAthero", ANR-13-LAB1-0005-01 "FucoChem" and ANR-13-RPIB-0006 "FucoThrombo".

\section{Author Contributions}

Conceived and designed the experiments: CC, LD, DLG, FC, FR, PS. Performed physico-chemical experiments: PS, NB, LP, MM. Performed radiolabeling: FAS, RBA, FR. Designed, performed and analyzed animal experiments: FR, LL. Contributed materials/reagents: LR, GP. Contributed to writing the manuscript: PS, FC, FR, DL, CC.

\section{Conflicts of Interest}

L.R. and G.P. are employees of Algues \& Mer, Kernigou, F-29242, Ouessant, France. The other authors declare no competing financial interest.

\section{References}

1. Finegold, J.A.; Asaria, P.; Francis, D.P. Mortality from ischaemic heart disease by country, region, and age: Statistics from World Health Organisation and United Nations. Int. J. Cardiol. 2013, 168, 934-945.

2. Lalatonne, Y.; Paris, C.; Serfaty, J.M.; Weinmann, P.; Lecouvey, M.; Motte, L. Bis-phosphonates-Ultra small superparamagnetic iron oxide nanoparticles: A platform towards diagnosis and therapy. Chem. Commun. 2008, 22, 2553-2555.

3. Corr, S.A.; O’Byrne, A.; Gun'ko, Y.K.; Ghosh, S.; Brougham, D.F.; Mitchell, S.; Volkov, Y.; Prina-Mello, A. Magnetic-fluorescent nanocomposites for biomedical multitasking. Chem. Commun. 2006, 43, 4474-4476.

4. Masotti, A.; Pitta, A.; Ortaggi, G.; Corti, M.; Innocenti, C.; Lascialfari, A.; Marinone, M.; Marzola, P.; Daducci, A.; Sbarbati, A.; et al. Synthesis and characterization of polyethylenimine-based iron oxide composites as novel contrast agents for MRI. Magn. Resonance Mater. Phys. Biol. Med. 2009, 22, 77-87.

5. Petri-Fink, A.; Chastellain, M.; Juillerat-Jeanneret, L.; Ferrari, A.; Hofmann, H. Development of functionalized superparamagnetic iron oxide nanoparticles for interaction with human cancer cells. Biomaterials 2005, 26, 2685-2694.

6. Xie, J.; Wang, J.; Niu, G.; Huang, J.; Chen, K.; Li, X.; Chen, X. Human serum albumin coated iron oxide nanoparticles for efficient cell labeling. Chem. Commun. 2010, 46, 433-435.

7. Tsourkas, A.; Shinde-Patil, V.R.; Kelly, K.A.; Patel, P.; Wolley, A.; Allport, J.R.; Weissleder, R. In vivo imaging of activated endothelium using an anti-VCAM-1 magnetooptical probe. Bioconjug. Chem. 2005, 16, 576-581. 
8. Gao, F.; Cai, Y.; Zhou, J.; Xie, X.; Ouyang, W.; Zhang, Y.; Wang, X.; Zhang, X.; Wang, X.; Zhao, L.; Tang, J. Pullulan acetate coated magnetite nanoparticles for hyper-thermia: Preparation, characterization and in vitro experiments. Nano Res. 2010, 3, 23-31.

9. Molday, R.S.; MacKenzie, D. Immunospecific ferromagnetic iron-dextran reagents for the labeling and magnetic separation of cells. J. Immunol. Methods 1982, 52, 353-367.

10. Kievit, F.M.; Veiseh, O.; Bhattarai, N.; Fang, C.; Gunn, J.W.; Lee, D.; Ellenbogen, R.G.; Olson, J.M.; Zhang, M. PEI-PEG-Chitosan-Copolymer-Coated iron oxide nanoparticles for safe gene delivery: Synthesis, complexation, and transfection. Adv. Funct. Mater. 2009, 19, 2244-2251.

11. Pomin, V.H. Fucanomics and galactanomics: Current status in drug discovery, mechanisms of action and role of the well-defined structures. Biochim. Biophys. Acta Gen. Subj. 2012, 1820, 1971-1979.

12. Berteau, O.; Mulloy, B. Sulfated fucans, fresh perspectives: Structures, functions, and biological properties of sulfated fucans and an overview of enzymes active toward this class of polysaccharide. Glycobiology 2003, 13, 29R-40R.

13. Ustyuzhanina, N.E.; Bilan, M.I.; Ushakova, N.A.; Usov, A.I.; Kiselevskiy, M.V.; Nifantiev, N.E. Fucoidans: Pro- or antiangiogenic agents? Glycobiology 2014, doi:10.1093/glycob/cwu063.

14. Kwak, J.Y. Fucoidan as a marine anticancer agent in preclinical development. Mar. Drugs 2014, 12, 851-870.

15. Blondin, C.; Fischer, E.; Boisson-Vidal, C.; Kazatchkine, M.D.; Jozefonvicz, J. Inhibition of complement activation by natural sulfated polysaccharides (fucans) from brown seaweed. Mol. Immunol. 1994, 31, 247-253.

16. Tissot, B.; Daniel, R. Biological properties of sulfated fucans: The potent inhibiting activity of algal fucoidan against the human complement system. Glycobiology 2003, 13, 29G-30G.

17. Bachelet, L.; Bertholon, I.; Lavigne, D.; Vassy, R.; Jandrot-Perrus, M.; Chaubet, F.; Letourneur, D. Affinity of low molecular weight fucoidan for P-selectin triggers its binding to activated human platelets. Biochim. Biophys. Acta Gen. Subj. 2009, 1790, 141-146.

18. Rouzet, F.; Bachelet-Violette, L.; Alsac, J.M.; Suzuki, M.; Meulemans, A.; Louedec, L.; Petiet, A.; Jandrot-Perrus, M.; Chaubet, F.; Michel, J.B.; et al. Radiolabeled fucoidan as a P-Selectin targeting agent for in vivo imaging of platelet-rich thrombus and endothelial activation. J. Nucl. Med. 2011, 52, 1433-1440.

19. Suzuki, M.; Bachelet-Violette, L.; Rouzet, F.; Beilvert, A.; Autret, G.; Maire, M.; Menager, C.; Louedec, L.; Choqueux, C.; Saboural, P.; et al. Ultrasmall superparamagnetic iron oxide nanoparticles coated with fucoidan for molecular MRI of intraluminal thrombus. Nanomedicine 2014, doi:10.2217/nnm.14.51.

20. Bachelet-Violette, L.; Silva, A.K.A.; Maire, M.; Michel, A.; Brinza, O.; Ou, P.; Ollivier, V.; Nicoletti, A.; Wilhelm, C.; Letourneur, D.; et al. Strong and specific interaction of ultra small superparamagnetic iron oxide nanoparticles and human activated platelets mediated by fucoidan coating. RSC Adv. 2014, 4, 4864-4871.

21. Bernardi, G.; Springer, G.F. Properties of highly purified fucan. J. Biol. Chem. 1962, 237, 75-80.

22. Patankar, M.S.; Oehninger, S.; Barnett, T.; Williams, R.L.; Clark, G.F. A revised structure for fucoidan may explain some of its biological activities. J. Biol. Chem. 1993, 268, 21770-21776. 
23. Choi, J.; Gu Lee, S.; Jong Han, S.; Cho, M.; Cheon Lee, P. Effect of gamma irradiation on the structure of fucoidan. Radiat. Phys. Chem. 2014, 100, 54-58.

24. Ale, M.T.; Maruyama, H.; Tamauchi, H.; Mikkelsen, J.D.; Meyer, A.S. Fucoidan from Sargassum sp and Fucus vesiculosus reduces cell viability of lung carcinoma and melanoma cells in vitro and activates natural killer cells in mice in vivo. Int. J. Biol. Macromol. 2011, 49, 331-336.

25. Ale, M.T.; Meyer, A.S. Fucoidans from brown seaweeds: An update on structures, extraction techniques and use of enzymes as tools for structural elucidation. RSC Adv. 2013, 3, 8131.

26. Pomin, V.H.; Mourão, P.A.S. Structure, biology, evolution, and medical importance of sulfated fucans and galactans. Glycobiology 2008, 18, 1016-1027.

27. Kylin, H. Zur Biochemie der Meeresalgen. Z. Für Physiol. Chem. 1913, 83, 171-197.

28. Kloareg, B.; Quatrano, R. S. Structure of the cell walls of marine algae and ecophysiological functions of the matrix polysaccharides. In Oceanography and Marine Biology; Barnes, M., Ed.; Aberdeen University Press: Aberdeen, UK, 1988; Volume 26, pp. 259-315.

29. Chaubet, F.; Chevolot, L.; Jozefonvicz, J.; Durand, P.; Boisson-Vidal, C. Relationships between chemical characteristics and anticoagulant activity of low molecular weight fucans from marine algae. In Bioactive Carbohydrate Polymers; Paulsen, B.S., Ed.; Springer: Heidelberg, Germany, 2000; Volume 44, pp. 59-84.

30. Nishino, T.; Nishioka, C.; Ura, H.; Nagumo, T. Isolation and partial characterization of a novel amino sugar-containing fucan sulfate from commercial fucus vesiculosus fucoidan. Carbohydr. Res. 1994, 255, 213-224.

31. Chizhov, A.O.; Dell, A.; Morris, H.R.; Haslam, S.M.; McDowell, R.A.; Shashkov, A.S.; Nifantiev, N.E.; Khatuntseva, E.A.; Usov, A.I. A study of fucoidan from the brown seaweed Chorda filum. Carbohydr. Res. 1999, 320, 108-119.

32. Chevolot, L.; Mulloy, B.; Ratiskol, J.; Foucault, A.; Colliec-Jouault, S. A disaccharide repeat unit is the major structure in fucoidans from two species of brown algae. Carbohydr. Res. 2001, 330, 529-535.

33. Bilan, M.I.; Grachev, A.A.; Ustuzhanina, N.E.; Shashkov, A.S.; Nifantiev, N.E.; Usov, A.I. Structure of a fucoidan from the brown seaweed Fucus evanescens C.Ag. Carbohydr. Res. 2002, 337, 719-730.

34. Mabeau, S.; Kloareg, B. Isolation and analysis of the cell walls of brown algae: Fucus spiralis, F. ceranoides, F. vesiculosus, F. serratus, Bifurcaria bifurcata and Laminaria digitata. J. Exp. Bot. 1987, 38, 1573-1580.

35. Leite, E.L.; Medeiros, M.G.L.; Rocha, H.A.O.; Farias, G.G.M.; da Silva, L.F.; Chavante, S.F.; de Abreu, L.D.; Dietrich, C.P.; Nader, H.B. Structure and pharmacological activities of a sulfated xylofucoglucuronan from the alga Spatoglossum schroederi. Plant Sci. 1998, 132, 215-228.

36. Daniel, R.; Berteau, O.; Chevolot, L.; Varenne, A.; Gareil, P.; Goasdoue, N. Regioselective desulfation of sulfated L-fucopyranoside by a new sulfoesterase from the marine mollusk Pecten maximus-Application to the structural study of algal fucoidan (Ascophyllum nodosum). Eur. J. Biochem. 2001, 268, 5617-5626.

37. Chevolot, L.; Foucault, A.; Chaubet, F.; Kervarec, N.; Sinquin, C.; Fisher, A.M.; Boisson-Vidal, C. Further data on the structure of brown seaweed fucans: Relationships with anticoagulant activity. Carbohydr. Res. 1999, 319, 154-165. 
38. Rioux, L.E.; Turgeon, S.L.; Beaulieu, M. Characterization of polysaccharides extracted from brown seaweeds. Carbohydr. Polym. 2007, 69, 530-537.

39. Kulkarni, P.V.; Parkey, R.W.; Wilson, J.E.; Lewis, S.E.; Buja, L.M.; Bonte, F.J.; Willerson, J.T. Modified Technetium-99m Heparin for the Imaging of Acute Experimental Myocardial Infarcts. J. Nucl. Med. 1980, 21, 117-121.

40. Dische, Z. New Color Reactions for Determination of Sugars in Polysaccharides. In Methods of Biochemical Analysis; Glick, D., Ed.; John Wiley \& Sons, Inc.: Hoboken, NJ, USA, 1955; Volume 2, pp. 313-358.

41. Bitter, T.; Muir, H.M. A modified uronic acid carbazole reaction. Anal. Biochem. 1962, 4, 330-334.

42. Gustafsson, L. Determination of ultramicro amounts of sulphate as methylene blue-II The reduction. Talanta 1960, 4, 236-243.

43. Kuban, V.; Dasgupta, P.K.; Marx, J.N. Nitroprusside and methylene blue methods for silicone membrane differentiated flow injection determination of sulfide in water and wastewater. Anal. Chem. 1992, 64, 36-43.

44. Spies, H.; Pietzsch, H.J. Stannous Chloride in the Preparation of 99mTc Pharmaceuticals. In Technetium-99m Pharmaceuticals; Zolle, I., Ed.; Springer Berlin: Heidelberg, Germany, 2007; pp. 59-66.

45. Saffari, H.; Krstyen, J.J.; Gonzalez, C.; Clayton, F.C.; Leiferman, K.M.; Gleich, G.J.; Peterson, K.A.; Pease, L.F. 99mTechnetium-labeled heparin: A new approach to detection of eosinophilic esophagitis-associated inflammation. J. Allergy Clin. Immunol. 2013, 132, 1446-1448.

46. Dass, R.S.; Singh, A.K.; Chauhan, U.P. Development of a dextran kit for labelling with 99mTc and its evaluation for lymphoscintigraphy. Nucl. Med. Biol. 1993, 20, 701-706.

(C) 2014 by the authors; licensee MDPI, Basel, Switzerland. This article is an open access article distributed under the terms and conditions of the Creative Commons Attribution license (http://creativecommons.org/licenses/by/3.0/). 